\title{
A majority of rural emergency departments in the province of Quebec use point-of-care ultrasound: a cross-sectional survey
}

\author{
Pierre Léger ${ }^{1}$, Richard Fleet ${ }^{1,2^{*}}$, Julie Maltais- Giguère ${ }^{2}$, Jeff Plant ${ }^{3,4}$, Éric Piette ${ }^{5}$, France Légaré ${ }^{6}$ and Julien Poitras ${ }^{1,2}$
}

\begin{abstract}
Background: Point-of-care ultrasound (POCUS) can be used to provide rapid answers to specific and potentially life-threatening clinical questions, and to improve the safety of procedures. The rate of POCUS access and use in Canada is unclear. The objective of this study was to examine access to POCUS and potential barriers/facilitators to its use among rural physicians in Quebec.
\end{abstract}

Methods: This descriptive cross-sectional study used an online survey. The 30-item questionnaire is an adapted and translated version of a questionnaire used in a prior survey conducted in rural Ontario, Canada. The questionnaire was pre-tested for clarity and relevance. The survey was sent to non-locum physicians working either full- or part-time in rural emergency departments (EDs) $(n=206)$. All EDs were located in rural and small towns and provided 24/7 medical coverage with acute care hospitalization beds.

Results: In total, 108 surveys were completed (participation rate $=52.4 \%$ ). Of the individuals who completed surveys, ninety-three percent were family physicians, and seven percent had Canadian College of Family Physicians - Emergency Medicine (CCFP-EM) certification. The median number of years of practice was seven. A bedside ultrasound device was available in $95 \%$ of rural EDs; $75.9 \%$ of physicians reported using POCUS on a regular basis. The most common indications for POCUS use were to rule out abdominal aortic aneurysm (70.4 \%) and to evaluate presence of free fluid in trauma and intrauterine pregnancy (60 \%). The most common reason (73 \%) for not using POCUS was limited access to POCUS training programs. Over $40 \%$ of POCUS users received training in POCUS during medical school or residency. Sixty-four percent received training from the Canadian Emergency Ultrasound Society, $13 \%$ received training from the Canadian Association of Emergency Physicians, and $23 \%$ were trained in another course. Finally, $95 \%$ of respondents reported that POCUS skills are essential for rural ED practice.

Conclusions: POCUS use in rural EDs in the province of Quebec appears to be relatively widespread. Access to training programs is a barrier to greater use.

\section{Background}

Emergency department (ED) point-of-care ultrasound (POCUS) can be performed rapidly at patients' bedside. POCUS results can respond to specific and potentiallylife threatening clinical questions. Furthermore, compliance with POCUS procedures improves patient safety $[1,2]$. POCUS is a safe and relatively low-cost

\footnotetext{
* Correspondence: rfleet@videotron.ca

'Department of Family and Emergency Medicine, Université Laval, Quebec City, QC, Canada

${ }^{2}$ Research Centre, Emergency Medicine Laval University - CHAU Hôtel-Dieu de Lévis Hospital, 143 Wolfe Street, Lévis, QC G6V 3Z1, Canada

Full list of author information is available at the end of the article
}

technology [1]. For the focused scans often used in emergency practice, the learning curve for POCUS is not considered steep $[1,2]$. Its use improves diagnostic accuracy and timely management of selected urgent conditions [3]. Many international medical associations have already endorsed POCUS as a standard of care $[4,5]$ and recommend training in POCUS as part of residency training and continuing medical education [6-9].

In 2006 and 2012, the Canadian Association of Emergency Physicians (CAEP) highlighted the importance of providing 24/7 access to POCUS in EDs nationwide $[10,11]$. Despite the CAEP position on POCUS and its potential benefits, the statistics on actual use of POCUS in 
Canada are not clear $[10,11]$. The literature in this area suggests that POCUS is increasingly taught and used in academic EDs in Canada [12, 13]. However, with the exception of one recent study conducted in Ontario [14], the use of POCUS in rural EDs has not been studied.

Rural EDs have limited local access to advanced imaging techniques such as computed tomography (CT) and formal ultrasound, and inter-facility transfers are often required to pursue diagnostic evaluations [15-18]. However, inter-facility transfer processes in rural and isolated areas can be costly, time-consuming and risky for patients and paramedics $[19,20]$. POCUS has the potential to improve triage of patients requiring emergency inter-facility transfers, and to consequently improve access to timely care [1].

According to Flynn et al. [14], POCUS is not used to full potential in rural settings in Ontario. Flynn et al. [14] reported that only $60 \%$ of their sample of 200 rural physicians had access to a bedside ultrasound device. Furthermore, only $44.4 \%$ of physicians reported having the necessary knowledge or skills to perform POCUS. However, over $70 \%$ of the sample stated that POCUS competence was an essential skill for the practice of rural emergency medicine [14]. The Flynn et al. study is recent and provides essential information; however, the low response rate $(28.4 \%)$ limits interpretation of the results, and further study of POCUS use in rural settings is warranted. The present study was designed to examine access to POCUS and potential barriers/facilitators to POCUS use among rural emergency physicians in Quebec.

\section{Methods}

This descriptive cross-sectional study used an online survey (SurveyMonkey Inc, Palo Alto, California, USA). The study was added $a$ posteriori to the ongoing Quebec Rural Emergency Department Project [15]. The research ethics committee of the "Centre de Santé et des Services Sociaux (CSSS)" Alphonse-Desjardins hospital approved the amendment to the project. The study's objectives and the procedure were explained in the questionnaire. All respondents agreed to participate freely to the study by checking "yes" on the questionnaire.

\section{Selection of rural EDs and participants}

Complete details on the definition of rural EDs and selection methods for the EDs included in the present study are provided elsewhere [15]. In brief, the Canadian Healthcare Facilities Guide was used to identify rural EDs providing 24/7 physician coverage and located in hospitals with acute-care hospitalization beds. We used Statistics Canada's definition of rural and small towns to select communities to participate [21]. In total, 26 EDs met our inclusion criteria; of the 26, 19 agreed to participate in the study. To be eligible to participate, emergency physicians had to work for at least one of the 19 participating rural EDs.

\section{Data collection}

The survey used in the Flynn et al. study [14] was translated into French, modified and adapted as per the literature on the subject. In compliance with recent survey design guidelines [22], the questionnaire was pre-tested for question clarity and item relevance on a convenience sample of ten emergency medicine residents from the Laval University Royal College of Physicians and Surgeons of Canada (FRCP). Individuals in the convenience sample had advanced training in POCUS, and their suggestions were used to modify and clarify the survey questionnaire. The final version of the survey included 30 questions. Eleven of the 30 questions pertained to socio-demographic information, including age, gender, level of medical training, year of graduation, number of years in practice, number of years practicing in the present community and distance to the nearest referral center. Two questions inquired about the availability of advanced imaging services. Seventeen questions addressed access and use of POCUS, including frequency of POCUS use, reason for POCUS use, and training in POCUS in medical school. The survey consisted of yes or no questions, multiple-choice questions and open-ended short answer questions. Questionnaire completion time was estimated to be less than ten minutes.

Data was collected between April 2013 and August 2013. The online survey (Survey Monkey software) was sent via e-mail to all part-time and full-time emergency staff practicing $(n=206)$ in the 19 selected rural EDs. To enhance participation, four automated reminder e-mails were sent at two-week intervals. Emergency department chairs were subsequently contacted by phone or e-mail and asked to remind colleagues of the study and to encourage participation.

\section{Statistical analysis}

Data was imported into Microsoft Excel from the Survey Monkey software. We used simple descriptive analysis and calculated percentage and median. Data were analyzed with Excel (Microsoft Office) 2010.

\section{Results}

In total, 108 of 206 eligible physicians participated in this study (participation rate $=52.4 \%$ ) Respondents' descriptive characteristics are reported in Table 1. Briefly, $54.6 \%$ of respondents were male, median age was 37 years and median year of graduation was 2006.

Table 2 presents the general characteristics of the EDs in the study. In summary, $95.4 \%$ of respondents reported access to a POCUS device. Most EDs had over 10,000 
Table 1 General characteristics of physicians

\begin{tabular}{ll}
\hline Characteristics & Median or (\%) $n=108$ \\
\hline Gender $(n[\%])$ & $59(54.6)$ \\
Male & $49(45.4)$ \\
Female & \\
Age & $37(25-62)$ \\
$\quad$ Median (range) & \\
Medical training $n$ (\%) & $100(92.6)$ \\
CCFP & $7(6.5)$ \\
CCFP (EM) & $0(0)$ \\
FRCP (EM) & $1(0.9)$ \\
Other & \\
Years in practice & $7(<1-34)$ \\
Median (range) & \\
Years practicing in present community & $5.5(<1-34)$ \\
Median (range) & \\
Year of graduation & $2006(1979-2013)$ \\
Median (range) & \\
Distance to nearest referral center (km) $(n[\%])$ & $10(9.3)$ \\
$<50$ & $20(18.5)$ \\
$50-90$ & $36(33.3)$ \\
$100-199$ & $42(38.9)$ \\
$>200$ & \\
\hline
\end{tabular}

No. number, CCFP certification in Family Medicine from the College of Family Physicians of Canada, CCFP (EM) certificate in special competence in emergency medicine from the College of Family Physicians of Canada, FRC(EM) Royal College of Physicians and Surgeons of Canada (Emergency Medicine Board Certification)

visits per year. The majority of participating EDs (78 \%) had local 24/7 access to CT scanners.

Seventy-six percent of physicians reported POCUS use (Table 3). The two most common reasons for use were to rule out an abdominal aortic aneurysm (70.4\%) and to verify the presence of free fluid in the abdomen (70.4\%). Seventy-three percent of physicians who reported not using POCUS cited lack of training as the primary reason. Over $40 \%$ of POCUS users reported having been trained in POCUS during their academic medical training. Sixty-four percent of trained users received training through the POCUS course offered by the Canadian Emergency Ultrasound Society (CEUS). Finally, half of non-trained physicians cited long waiting lists as the primary reason for not obtaining training in POCUS.

\section{Discussion}

The results of this study suggest that some of the CAEP's recommendations for POCUS use have been implemented in rural EDs in Quebec. They include availability of the device, scope of practice and training. Almost all rural physicians in Quebec EDs have access
Table 2 Rural ED characteristic

\begin{tabular}{ll}
\hline Variables & $n(\%)$ \\
\hline ED shifts per year & \\
$<40$ & $5(4.6)$ \\
$41-80$ & $32(29.6)$ \\
$81-120$ & $38(35.2)$ \\
$121-160$ & $24(22.2)$ \\
$>160$ & $9(8.3)$ \\
ED volume, patients per year & \\
$<5,000$ & $13(12.0)$ \\
$5,000-9,999$ & $13(12.0)$ \\
$10,000-14,000$ & $25(23.1)$ \\
15,000-19,000 & $22(20.4)$ \\
$>$ 20,000 & $35(32.4)$ \\
CT scanner in hospital (24/7 access) & \\
Yes & $85(78.7)$ \\
No & $23(21.3)$ \\
Ultrasonography in hospital (performed by radiologist) & \\
Yes, every day & $17(15.7)$ \\
Yes, sometimes & $80(74.1)$ \\
No & $11(10.2)$ \\
POCUS device available in ED & \\
Yes & $103(95.4)$ \\
\hline & $5(4.6)$ \\
\hline
\end{tabular}

No. number, ED emergency department, $C T$ computed tomography, POCUS point of care ultrasound

to a bedside ultrasound device, and a majority of physicians reported using it. The reported rates of POCUS access and use are significantly higher than the rates reported in the Flynn et al. study [14], in which only $60.6 \%$ of rural physicians had access to a POCUS device in the ED, and only $44.4 \%$ were trained to use it. One possible explanation for the inconsistent findings is that the Flynn et al. study [14] was conducted in 2010. A recent increase in POCUS training initiatives in residency programs may have contributed to increased use by new graduates over the past three years. This hypothesis is supported by the finding that rural physicians in Quebec were younger (median age 37 vs. 49) and were more likely to be recent graduates (average of 7 versus 15 years of clinical experience) than were Ontario physicians. Flynn et al. also reported that newer graduates reported greater POCUS use than did more experienced physicians [14].

It is likely that the number of emergency physicians using POCUS in Canada will continue to increase. As per Kim et al. [12], nearly all Canadian Emergency Medicine Programs now include POCUS training in their curriculum. All Royal College emergency medicine 
Table 3 Physician POCUS use

\begin{tabular}{l} 
Variables \\
\hline Use POCUS \\
Yes \\
No \\
Frequency of POCUS use \\
$<1$ per week \\
Once per ED shift \\
$>1$ per ED shift \\
Never \\
Reasons for POCUS use \\
Ruling out AAA \\
Ruling in intrauterine pregnancy \\
Ruling out peritoneal free fluid \\
Ruling out pericardial effusion \\
Central line placement \\
Do not use POCUS \\
Other \\
Do not perform POCUS because of ... ( $n=37$ ) \\
Lack of training \\
Difficulty maintaining skills \\
Lack of need \\
Oost
\end{tabular}

Training in POCUS during medical school

Yes

No

Where were you trained in POCUS? $(n=86)$

CAEP

CEUS

Other

Difficulty obtaining POCUS training because... $(n=52)$

Long waiting list

Distance to training center

Other

POCUS is a skill that an EP should have

Strongly agree

Agree

Neither agree nor disagree

Disagree

Strongly disagree

POCUS is a skill that a rural EP should have

Strongly agree

Agree

Neither agree nor disagree

Table 3 Physician POCUS use (Continued)

\begin{tabular}{ll}
\hline Disagree & $0(0.0)$ \\
Strongly disagree & $0(0.0)$ \\
Should pay for POCUS training ... & \\
RAMQ & $51(47.2)$ \\
Physician & $34(31.5)$ \\
Hospital & $15(13.9)$ \\
Community & $0(0.0)$ \\
Other & $8(7.4)$ \\
Should pay for the POCUS device ... & \\
RAMQ & $11(10.2)$ \\
Physician & $0(0.0)$ \\
Hospital & $94(87.0)$ \\
Community & $0(0.0)$ \\
Other & $3(2.8)$ \\
\hline AAA abdomil aotic antsm, CAEP Canndan Assocition of Emerg
\end{tabular}

AAA abdominal aortic aneurysm, CAEP Canadian Association of Emergency Physicians, CEUS Canadian Emergency Ultrasound Society, EP emergency physician, RAMQ Régie de l'assurance maladie du Québec

programs and $88 \%$ of Certificate in Special Competence 27 (73.0) in Emergency Medicine (CFPC-EM) programs include 12 (32.4) training in POCUS [9].

Nevertheless, we are surprised by the considerable access to and use of POCUS in Quebec's rural EDs at this time. Future studies are warranted to examine POCUS use in rural EDs in other Canadian provinces. To date, only a few studies have examined POCUS use 44 (40.7) in urban settings [23-25]. Comparisons between urban 64 (59.3) and rural use would be of interest, particularly in the context of reported increased use of CT scanners in academic centers [26].

$11(12.8)$

$55(64.0)$

$20(23.2)$

$26(50.0)$

$22(42.3)$

$14(26.9)$

$72(66.7)$

$31(28.7)$

$5(4.6)$

$0(0.0)$

$0(0.0)$

$67(62.0)$

$36(33.3)$

$5(4.6)$

\section{Strengths and limitations}

Although the $52 \%$ participation rate in the current constitutes a limitation, such rates are commonly reported rate in online surveys of physicians [22, 27]. Furthermore, the participation rate in the present study is significantly higher than the $28 \%$ response rate reported in the only other published report on POCUS use among Canadian rural physicians [14]. The possibility of response bias from enthusiastic POCUS users cannot be ruled out, and must be taken into consideration in interpretation of the results. The study's design did not allow us to gather data allowing comparisons between responders and non-responders, nor did we inquire about reasons for non-participation. Some of our e-mail invitations may have been lost in junk e-mail folders or sent to an incorrect address. Physician vacation or leaves of absence may have further decreased the participation rate. Our limited response rate despite multiple automated e-mail reminders and personal contacts with ED chairs may 
reflect the inherent challenges of conducting research in remote and non-academically affiliated centres. Although the survey questions were straightforward and the translated version was pre-tested on francophone residents, we cannot exclude the possibility that some elements were lost in translation. Finally, this study measured self-reported POCUS use; quality of POCUS use and impact on patient care were not determined.

The primary strength of this study is that the sample was composed of physicians working in hospitals in confirmed rural facilities participating in an ongoing study of every rural ED in Quebec. Although Quebec is Canada's second largest province, future studies are required to determine actual POCUS use in rural settings across Canada and in other countries.

\section{Conclusion}

POCUS use in rural EDs in the province of Quebec seems to be widespread and perhaps more common than in the province of Ontario. Improving access to training programs for rural emergency physicians may further increase POCUS use. Finally, further studies should explore CAEP recommendations that were not included in this study, such as leadership, self-governance, documentation, quality improvement, continuing medical education and research.

\section{Abbreviations}

CAEP: Canadian Association of Emergency Physicians; CCFP-EM: Canadian College of Family Physicians - Emergency Medicine; CEUS: Canadian Emergency Ultrasound Society; CSSS: Centre de Santé et des Services Sociaux; CT: computed tomography; ED: Emergency Departments; FRCP: Royal College of Physicians and Surgeons of Canada; POCUS: point-of-care ultrasound.

\section{Competing interests}

The authors declare no financial or other competing interest related to the submission of this manuscript. The manuscript represents original work and is not being considered for publication elsewhere. The research project was supported in part by a Junior 1 Clinician-Scientist award from the FRQS (RF), the Research Chair Emergency Medicine Laval University - CHAU Hôtel-Dieu de Lévis Hospital and the Hôtel-Dieu de Lévis Hospital research fund.

\section{Authors' contributions}

$\mathrm{PL}$ reviewed the literature, helped design the study and collected and analyzed data. Along with RF, he drafted the first version of the manuscript. RF was responsible for the overall study design and implementation. He supervised data analyses, and drafted the initial manuscript and its revised versions. JM-G coordinated the study, and participated in data collection and revision of the manuscripts. JP, ÉP and JP contributed to the literature review and to revisions of the manuscript. FL contributed to the study design and to the revision of the final manuscript. All authors read and approved the final version of the manuscript.

\section{Acknowledgements}

We wish to thank all of the rural emergency physicians and emergency department chairs in the province of Quebec for participating in this study. We also thank Fatoumata Korika Tounkara for her help in revising and formatting the manuscript.

\section{Author details}

'Department of Family and Emergency Medicine, Université Laval, Quebec City, QC, Canada. ${ }^{2}$ Research Centre, Emergency Medicine Laval University CHAU Hôtel-Dieu de Lévis Hospital, 143 Wolfe Street, Lévis, QC G6V 3Z1, Canada. ${ }^{3}$ Faculty of Medicine, University of British Columbia, Vancouver, BC, Canada. ${ }^{4}$ Department of Emergency Medicine, Penticton Regional Hospital, Penticton, BC, Canada. ${ }^{5}$ Department of Family and Emergency Medicine, Université de Montréal; Department of Emergency Medicine, Hôpital du Sacré-Coeur de Montréal, Montréal, QC, Canada. ${ }^{6}$ Department of Family Medicine and Emergency Medicine, Knowledge Transfer and Health Technology Assessment of the CHUQ Research Centre (CRCHUQ, Unité de Recherche Évaluative, Université Laval; Implementation of Shared Decision Making in Primary Care, Quebec City, QC, Canada.

Received: 27 March 2014 Accepted: 7 December 2015

Published online: 11 December 2015

\section{References}

1. Arienti V, Camaggi V. Clinical applications of bedside ultrasonography in internal and emergency medicine. Intern Emerg Med. 2011;6(3):195-201.

2. Moore $\mathrm{CL}$, Copel JA. Point-of-care ultrasonography. N Engl J Med. 2011:364(8):749-57.

3. Lyon M, Blaivas M, Brannam L. Use of emergency ultrasound in a rural ED with limited radiology services. Am J Emerg Med. 2005;23(2):212-4.

4. Thomas HA, Beeson MS, Binder LS, Brunett PH, Carter MA, Chisholm CD, et al. The 2005 model of the clinical practice of emergency medicine: the 2007 update. Acad Emerg Med. 2008;15(8):776-9.

5. ACEP. Emergency ultrasound guidelines. Ann Emerg Med. 2009;53(4):550-70

6. Heller MB, Mandavia D, Tayal VS, Cardenas EE, Lambert MJ, Mateer J, et al. Residency training in emergency ultrasound: fulfilling the mandate. Acad Emerg Med. 2002;9(8):835-9.

7. Mayo PH, Beaulieu Y, Doelken P, Feller-Kopman D, Harrod C, Kaplan A, et al. American College of Chest Physicians/La Societe de Reanimation de Langue Francaise statement on competence in critical care ultrasonography. Chest. 2009:135(4):1050-60.

8. Cholley BP, ICU ERTU. International expert statement on training standards for critical care ultrasonography. Intensive Care Med. 2011;37(7):1077-83.

9. Royal-College-of-Physicians-and-Surgeons-of-Canada. Objectives of Training in Emergency Medicine. 2011:1-23.

10. Henneberry J, Hanson A, Healey A, Hebert G, Ip U, Mensour M, et al. Use of point of care sonography by emergency physicians. Cjem. 2012:14(2):106-12

11. Socransky S. Emergency department targeted ultrasound: 2006 update. Cjem. 2006;8(3):170-4

12. Kim DJ, Theoret J, Liao MM, Hopkins E, Woolfrey K, Kendall JL. The current state of ultrasound training in canadian emergency medicine programs: perspectives from program directors. Acad Emerg Med. 2012;19(9):E1073-8.

13. Woo MY, Nussbaum C, Lee AC. Emergency medicine ultrasonography: national survey of family medicine-emergency medicine program directors. Can Fam Physician. 2009;55(10):1010-1. e1011-1014.

14. Flynn CJ, Weppler A, Theodoro D, Haney E, Milne WK. Emergency medicine ultrasonography in rural communities. Can J Rural Med. 2012;17(3):99-104.

15. Fleet $R$, Archambault $P$, Legare F, Chauny JM, Levesque JF, Ouimet $M$, et al. Portrait of rural emergency departments in Quebec and utilisation of the Quebec Emergency Department Management Guide: a study protocol. BMJ Open. 2013:3:4.

16. Fleet R, Audette LD, Marcoux J, Villa J, Archambault P, Poitras J. Comparison of access to services in rural emergency departments in Quebec and British Columbia. Cjem. 2013;15:1-12.

17. Fleet R, Poitras J, Maltais-Giguere J, Villa J, Archambault P. A descriptive study of access to services in a random sample of Canadian rural emergency departments. BMJ Open. 2013;3(11), e003876.

18. Rourke J, Kennard M. Emergency patient transfers from rural hospitals: a regional study. Cjem. 2001;3(4):296-301.

19. Sanddal N, Albert S, Hansen J, Kupas D. Contributing factors and issues associated with rural ambulance crashes: literature review and annotated bibliography. Prehosp Emerg Care. 2008;12(2):257-67.

20. Slattery D, Silver A. The hazards of providing care in emergency vehicles: an opportunity for reform. Prehosp Emerg Care. 2009:13(3):388-97.

21. du Plessis V, Beshiri R, Bollman RD, Clemenson H. Definitions of rural. In: Rural and Small Town Canada Analysis Bulletin, vol. 3. Ottawa: Statistics Canada; 2001. p. 1-17. Catalogue no.21-006-XIE. 
22. Burns KE, Duffett M, Kho ME, Meade MO, Adhikari NK, Sinuff T, et al. A guide for the design and conduct of self-administered surveys of clinicians. CMAJ. 2008;179(3):245-52.

23. Nagaraj G, Chu M, Dinh M. Emergency clinician performed ultrasound: availability, uses and credentialing in Australian emergency departments. Emerg Med Australas. 2010;22(4):296-300.

24. Stein JC, River G, Kalika I, Hebig A, Price D, Jacoby VL, et al. A survey of bedside ultrasound use by emergency physicians in California. J Ultrasound Med. 2009;28(6):757-63.

25. Talley BE, Ginde AA, Raja AS, Sullivan AF, Espinola JA, Camargo Jr CA. Variable access to immediate bedside ultrasound in the emergency department. West J Emerg Med. 2011;12(1):96-9.

26. Kocher KE, Meurer WJ, Fazel R, Scott PA, Krumholz HM, Nallamothu BK National trends in use of computed tomography in the emergency department. Ann Emerg Med. 2011:58(5):452-62. e453.

27. McLeod CC, Klabunde CN, Willis GB, Stark D. Health care provider surveys in the United States, 2000-2010: a review. Eval Health Prof. 2013:36(1):106-26.

Submit your next manuscript to BioMed Central and we will help you at every step:

- We accept pre-submission inquiries

- Our selector tool helps you to find the most relevant journal

- We provide round the clock customer support

- Convenient online submission

- Thorough peer review

- Inclusion in PubMed and all major indexing services

- Maximum visibility for your research

Submit your manuscript at www.biomedcentral.com/submit 